\title{
Modelos para parcerias entre governos e organizações da sociedade civil: análise comparativa de políticas de AIDS, assistência social e cultura no Brasil
}

\author{
Patrícia Maria Emerenciano de Mendonça 1 \\ Anny Karine de Medeiros ${ }^{2}$ \\ Edgilson Tavares de Araújo ${ }^{34}$ \\ 1 Universidade de São Paulo / Escola de Artes Ciências e Humanidades, São Paulo / SP - Brasil \\ 2 Fundação Getulio Vargas / Escola de Administração de Empresas de São Paulo, São Paulo / SP - Brasil \\ ${ }^{3}$ Universidade de Brasília / Centro de Estudos Avançados Multidisciplinares, Programa de Pós-Graduação em Desenvolvimento, \\ Sociedade e Cooperação Internacional, Brasília / DF - Brasil \\ ${ }^{4}$ Universidade Federal da Bahia, Salvador / BA - Brasil
}

\begin{abstract}
Este estudo contribui para a pesquisa sobre modelos de parceria, comparando diferentes áreas de política, testando as tipologias existentes e desenvolvendo novas formas de análise para o contexto brasileiro. A literatura sobre parcerias em relação ao setor sem fins lucrativos está focada na grande diversidade dessas organizações e nos tipos de relacionamento que estabelecem com o governo para a prestação de serviços. A maioria dos estudos empíricos sobre essa questão busca estabelecer categorias para as parcerias analisadas, geralmente comparando países ou áreas de políticas em um nível macro. Este estudo observa como os modelos de parceria ajudam a entender as diferenças entre áreas de políticas públicas, observando casos nas áreas de AIDS, assistência social e cultura no Brasil. A pesquisa introduziu variáveis de nível de campo e variáveis organizacionais para estabelecer diferenças mais explícitas entre os modelos e para identificar onde elas se sobrepõem. As diferenças entre os modelos ajudaram a testar as variáveis relacionais e usar os modelos de parceria para analisar as áreas de política pública no desenho de sua interação com as organizações. Quanto às implicações políticas deste estudo, ele fornece recomendações para avançar em uma agenda governamental sobre parcerias que podem combinar diretrizes gerais com particularidades relacionadas a cada área. Por fim, o estudo indica que as parcerias devem ser consideradas como instrumentos de política pública.
\end{abstract}

Palavras-chave: entidades sem fins lucrativos; parceiras; políticas sociais; colaboração.

\section{Modelos para alianzas entre gobiernos y organizaciones de la sociedad civil: análisis comparativo de políticas de SIDA, asistencia social y cultura en Brasil}

Este estudio contribuye a la investigación sobre modelos de alianzas al comparar diferentes áreas de políticas, probar las tipologías existentes y desarrollar nuevas formas de análisis para el contexto brasileño. La literatura sobre alianzas en relación con al sector de entidades sin fines de lucro se centra en la gran diversidad de estas instituciones y en los tipos de relación que establecen con el gobierno para brindar servicios. La mayoría de los estudios empíricos sobre este tema buscan establecer categorías para las alianzas analizadas, generalmente comparando países o áreas de políticas a nivel macro. Este estudio observa cómo los modelos de alianza ayudan a comprender las diferencias entre áreas de políticas públicas, observando casos en las áreas de SIDA, asistencia social y cultura en Brasil. La investigación introdujo variables de nivel de campo y variables organizativas para establecer diferencias más explícitas entre los modelos e identificar dónde se superponen. Las diferencias entre los modelos ayudaron a probar las variables relacionales y a usar los modelos de alianza para analizar las áreas de políticas públicas. En cuanto a las implicaciones políticas de este estudio, proporciona recomendaciones para avanzar en una agenda gubernamental sobre alianzas que pueden combinar directrices generales con particularidades relacionadas con cada área de política. Finalmente, el estudio indica que las alianzas deben considerarse como instrumentos de política pública.

Palabras clave: entidades sin fines de lucro; alianzas; políticas sociales; colaboración. 


\section{Models for government-nonprofits partnerships: a comparative analysis of policies for AIDS, social assistance and culture in Brazil}

This study contributes to the research on partnership models by comparing different policy areas, testing the existing typologies and developing new forms of analysis for the Brazilian context. The literature on partnerships regarding the nonprofit sector is focused on the great diversity of these organizations and the types of relationship they establish with the government to provide services. Most empirical studies on this issue seek to establish categories for the partnerships analyzed, usually comparing countries or policy areas on a macro level. This study observes how partnership models help to understand the differences among policy areas, observing cases in the areas of AIDS, social assistance, and culture in Brazil. The research introduced field level variables and organizational variables to establish clearer differences among the models, and to identify where they overlap. Differences among the models helped to test relational variables and use the partnership models to analyze the public policy areas on the interaction design with nonprofits. As for political implications, this study provides recommendations to advance in a governmental agenda on partnerships that can combine general guidelines with particularities related to each policy area. Finally, the study indicates that partnerships should be considered public policy instruments.

Keywords: nonprofit organizations; partnerships; social policies; collaboration.

\section{INTRODUÇÃO}

Estudos sobre parcerias entre governos e Organizações da Sociedade Civil (OSCs) foram concentrados em contextos europeus e norte-americanos, com poucos estudos em outros lugares. $\mathrm{Na}$ América Latina, faz-se necessário mais pesquisas para sistematizar esses modelos (Appe \& Layton, 2016; Peci, Figale, \& Sobral, 2011). O debate acadêmico sobre o tema no Brasil é disperso, focando questões como: regulação e controle, comparação entre vantagens e limitações de cada parte na prestação de serviços, sistematização de dados históricos sobre parcerias no nível federal e engajamento de profissionais de diversos setores (Alves \& Koga, 2006; Campos, 2008; Campos, Mendonça, \& Alves, 2012; Lopez \& Abreu 2014; Lopez, Lopes, Souto, \& Sant'Ana, 2014; Peci et al., 2011; Resende \& Teodosio, 2014; Salinas, 2013).

Diferentes tipos de parceria são estabelecidos entre as áreas de políticas públicas. A fim de criar uma estrutura analítica para parcerias, devemos levar em conta a natureza desse objeto como um espaço mutável de interações que combina diferentes lógicas organizacionais (Bode \& Brandsen, 2014; Brinkerhoff, 2002).

Este artigo pretende contribuir para esta agenda de pesquisa, comparando diferentes modelos de parceria, testando as tipologias existentes e desenvolvendo novas formas de análise para o contexto brasileiro. Três áreas de políticas públicas, AIDS, assistência social e cultura, foram selecionadas porque incluem parcerias no desenho de políticas públicas e programas, embora variem na forma de implementação local.

Neste estudo, a política pública não é entendida como sinônimo de instrumentos de política, embora exista uma forte relação metonímica entre eles (Boullosa, 2013). Parcerias não são políticas públicas per se, mas sim um instrumento de políticas públicas, ou seja, um dispositivo técnico e social que organiza as relações sociais específicas entre o poder público e os destinatários de suas ações em função de representações e de significados dos quais cada um é portador (Lascoumes \& Le Galés, 2012).

Esta introdução é seguida por uma seção detalhando a metodologia utilizada. A revisão teórica apresentada após a metodologia apresenta a literatura acadêmica sobre modelos e tipologias de 
parcerias, bem como descreve as categorias e níveis de análise utilizados. A quarta seção fornece uma breve visão geral das políticas de AIDS, assistência social e cultura (enfocando o programa Cultura Viva) em relação às parcerias entre governo e OSCs. A quinta seção apresenta uma sistematização e análise dos vários modelos encontrados, e discute as descobertas com base no modelo analítico desenvolvido na seção anterior.

As observações finais apontam para diferenças significativas de modelos entre áreas de políticas públicas, confirmando também que os modelos frequentemente se sobrepõem. No entanto, estes modelos são úteis na captura da complexidade e diversidade de parcerias, identificando diferenças e semelhanças entre áreas, sendo bons esquemas para testar proposições em diferentes contextos.

Pesquisas futuras focadas nesta agenda devem procurar combinar abordagens qualitativas e quantitativas com pesquisas sobre elementos específicos das parcerias e características das OSCs. Quanto às implicações em políticas públicas com base nos resultados encontrados nas parcerias analisadas em três áreas no Brasil, sugere-se avançar no debate sobre a agenda de parcerias para a prestação de serviços públicos, ressaltando a importância de diretrizes gerais e também das especificidades em cada área.

\section{METODOLOGIA}

O modelo analítico desenvolvido neste estudo baseou-se em revisão de literatura, utilizando como diretriz o trabalho de Doty e Glick (1994), que apresentam considerações importantes para diferenciar tipologias, com o propósito de construir teorias e propor hipóteses.

Segundo Doty e Glick (1994), as tipologias são bons esquemas para lidar com contextos diversos e complexos. São construções mais abstratas do que apenas classificações, pois devem ser objeto de rigorosos testes empíricos. As tipologias são compostas de conjuntos inter-relacionados de tipos ideais, cada uma representando uma combinação única de atributos que, acredita-se, influenciam os resultados. Nossa proposta de modelo analítico, contendo tipos ideais de parceria e seus atributos, é apresentada em detalhes na próxima sessão.

A fim de testar este modelo e fazer sugestões para desenvolvimentos futuros, esta pesquisa observou três diferentes e representativos casos de parcerias em políticas públicas nas áreas de AIDS, assistência social e cultura. Para tanto realizamos uma análise de múltiplos estudos de caso foi realizada, considerando que, conforme recomendado pela literatura, diferentes casos podem não ser diretamente comparáveis, mas é possível aprender a partir da análise conjunta deles (Stake, 1995).

As políticas públicas de AIDS e assistência social têm uma longa história de envolvimento com organizações sem fins lucrativos (Araújo, 2015a; Campos \& Mendonça, 2010). Na área da cultura, o programa analisado (Cultura Viva) foi criado em 2004 e foi considerado inovação enquanto política cultural e na forma como desenhou parcerias (Medeiros, 2013).

Este estudo considerou apenas o desenho da política pública, que ocorre no nível do governo central, não analisando a implementação no nível local. A análise em nível federal permite comparabilidade, mas limita as opções de detalhamento, uma vez que cada política/programa pode se desdobrar de forma diferente em contextos locais de implementação. Ao mesmo tempo, políticas e diretrizes formuladas no nível federal impactam especificamente a natureza dos relacionamentos em contextos locais de implementação, independentemente de quão heterogêneos e diversificados eles sejam. 
Como mencionado anteriormente, parcerias não são políticas públicas per se, mas sim instrumento de políticas públicas. Assim, os casos de parceria analisados mostram concepções complexas, que combinam diferentes instrumentos de políticas públicas para o fornecimento de bens e serviços públicos.

O escopo de tempo para a análise dos três casos foi limitado entre 2002 a 2015, que abrange o principal período de expansão de políticas sociais (e expansão de parcerias com OSCs) no Brasil, observando que a maioria das formas de prestação de serviços tinha algum grau de participação de OSCs.

Segundo Eisenhardt (1989), as abordagens de construção de teorias tipicamente combinam múltiplos métodos de coleta de dados, tais como a triangulação, que é uma maneira viável de fornecer uma comprovação mais forte de construtos e hipóteses (Eisenhardt, 1989). Seguindo esse direcionamento, os dados utilizados nesta pesquisa foram coletados por meio de bases de dados primárias e secundárias: revisão de literatura e documentos governamentais. Após uma primeira sistematização utilizando dados secundários, os dados primários foram utilizados para preencher as lacunas e triangular os resultados, obtidos por meio de entrevistas. Fomos capazes de conduzir cinco entrevistas em profundidade com gestores focais, três no Ministério da Saúde, um do Ministério do Desenvolvimento Social e um no Ministério da Cultura. Nesse sentido, o estudo foi baseado em extensos materiais existentes que foram reorganizados para seus fins, conforme mostrado no Quadro 1.

\section{QUADRO 1 MÉTODOS E FONTES PARA A COLETA DE DADOS}

\begin{tabular}{|c|c|c|c|}
\hline Métodos de coleta de dados & AIDS & Assistência Social & Cultura \\
\hline $\begin{array}{l}\text { Dados secundários de } \\
\text { projetos de pesquisa e } \\
\text { publicações anteriores }\end{array}$ & $\begin{array}{l}\text { Campos e Mendonça } \\
\text { (2010), Campos et al. } \\
\text { (2012), que utilizaram } \\
\text { abordagens qualitativas } \\
\text { baseadas em entrevistas, } \\
\text { análise de documentos e } \\
\text { descrições detalhadas de } \\
\text { casos. }\end{array}$ & $\begin{array}{l}\text { Araújo (2015a, 2015b, 2015c), } \\
\text { que utilizou pesquisa qualitativa } \\
\text { voltada para pessoas com } \\
\text { deficiência, com base em } \\
\text { entrevistas, grupo focal e } \\
\text { pesquisa participativa. }\end{array}$ & $\begin{array}{l}\text { Medeiros (2013), Medeiros, } \\
\text { Alves, e Farah (2015), que } \\
\text { utilizou abordagens qualitativas } \\
\text { e quantitativas com base } \\
\text { em entrevistas, análise } \\
\text { documental, levantamento } \\
\text { com } 31 \text { Pontos de Cultura } \\
\text { (Medeiros, 2013). }\end{array}$ \\
\hline $\begin{array}{l}\text { Principais referências } \\
\text { bibliográficas e } \\
\text { documentais descrevendo } \\
\text { cada área de política }\end{array}$ & $\begin{array}{l}\text { Teixeira (1997), Parker } \\
\text { (2003), Galvão (2000), } \\
\text { Brasil (2015). }\end{array}$ & $\begin{array}{l}\text { Stuchi, Paula, e Paz (2012), } \\
\text { Mestriner (2001), Brasil (2009), } \\
\text { Sposati (2008, 2009, 2012). }\end{array}$ & Turino (2009), Brasil (2013). \\
\hline $\begin{array}{l}\text { Dados primários - } \\
\text { Entrevistas realizadas entre } \\
\text { dezembro de } 2015 \text { e março } \\
\text { de } 2016\end{array}$ & $\begin{array}{l}\text { Ministério da Saúde } \\
\text { (Departamento de DST, } \\
\text { Aids e Hepatites Virais, } \\
\text { três gestores foram } \\
\text { entrevistados). }\end{array}$ & $\begin{array}{l}\text { Ministério do Desenvolvimento } \\
\text { Social (Departamento de Rede } \\
\text { de Assistência Social Privada } \\
\text { do SUAS - Sistema Único de } \\
\text { Assistência Social, um gestor } \\
\text { entrevistado). }\end{array}$ & $\begin{array}{l}\text { Ministério da Cultura } \\
\text { (Secretaria de Cidadania } \\
\text { e Diversidade Cultural, um } \\
\text { gerente entrevistado). }\end{array}$ \\
\hline
\end{tabular}

Fonte: Elaborado pelos autores. 


\subsection{Modelos de relacionamento entre governo e organizações da sociedade civil}

A perspectiva econômica que foca em falhas de mercado, governo e filantrópicas está entre as perspectivas teóricas que fornecem uma compreensão da relação entre governo e OSCs na provisão de bens públicos (Hansmann, 1987; Lipsky \& Smith 1990; Salamon, 1995; Young, 2000).

De acordo com Young (2000), existem três modelos para as relações governo-organizações sem fins lucrativos, o suplementar, complementar e modelos adversariais. No modelo suplementar, organizações sem fins lucrativos oferecem novas maneiras de fornecer serviços que o estado não oferece - geralmente estimulando a inovação. Quanto mais heterogêneas as preferências dos cidadãos por bens públicos, mais se espera que OSCs também ofertem tais serviços, como acontece na área da cultura e das artes.

Outros estudos baseados em uma perspectiva micro sociológica, notavelmente Coston (1998) e Najam (2000), são úteis para abranger formas mais diversas de relacionamento, destacando tanto o setor (o nível principal de análise das abordagens econômicas) quanto os níveis organizacionais.

Coston (1998) apresenta um modelo com oito tipologias de relações governo-OSCs, considerando o pluralismo institucional e as assimetrias de poder, desde a repressão à colaboração, como mostra a Figura 1.

\section{FIGURA $1 \quad$ TIPOLOGIAS DE RELAÇÕES OSCS-GOVERNO DE COSTON}

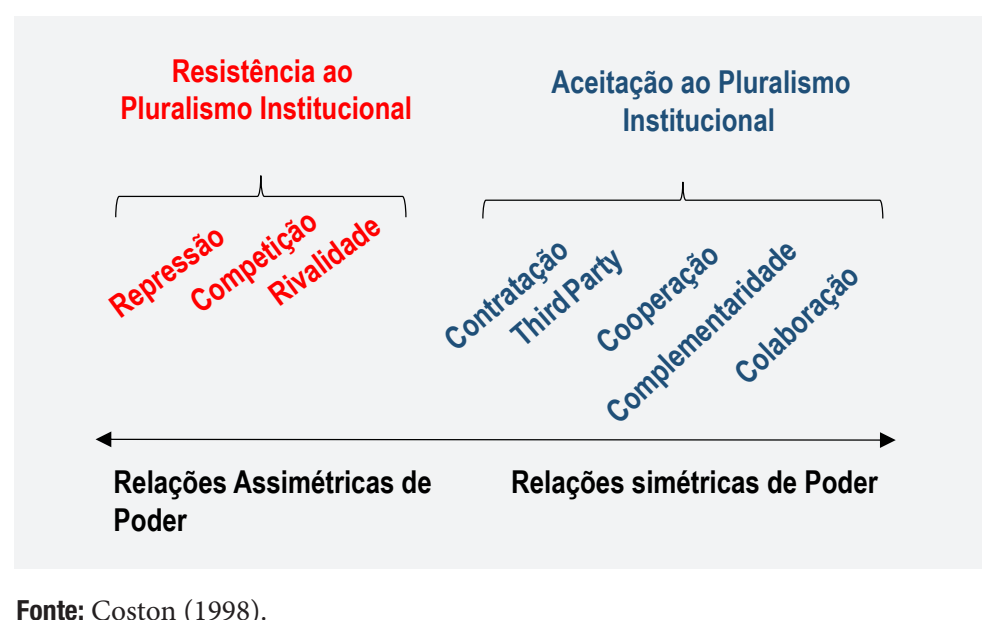

Argumentos econômicos para parcerias só se tornam válidos no modelo de Coston para os tipos de relacionamentos que aceitam o pluralismo institucional, enquanto tipos mais formalizados apresentam vantagens comparativas. Os tipos de relacionamento contratual e de terceiros (third party) são baseados em formalidade, enquanto cooperação, complementaridade e colaboração são tipos menos formalizados. Graus de formalização também podem aparecer em contextos que rejeitam pluralismo institucional, na forma de políticas públicas e regulamentações que restringem as atividades das OSCs, ou mesmo a sua existência. 
Coston (1998) e Young (2000) mencionam repetidamente os critérios de eficiência na contratação de OSCs como parceiras, sendo importante permitir um nível mínimo de competição entre elas ou mesmo a competição entre estas organizações e organizações com fins lucrativos ${ }^{1}$.

Referindo-se a Coston (1998), Najam (2000) defende uma simplificação nos tipos propostos, apontando a dificuldade em diferenciar alguns deles, como cooperação e colaboração. Além disso, a perfeita simetria de poder não é um pré-requisito para a cooperação. O comportamento cooperativo é alcançado porque nenhum dos atores considera suas intenções e ações desafiadas.

Quando Najam (2000) refere-se à cooperação, ele reconhece algumas expressões diferentes empregadas na literatura: cooperação, colaboração, co-produção, governança, de complementaridade, entre outros. Eles geralmente se referem a práticas tão diversas quanto metas comuns alinhadas, normas compartilhadas, comunicação aberta, esforços de coordenação, vantagens comparativas, compartilhamento de recursos, ação conjunta, termos também discutidos por Coston (1998), Young (2000) e Brinkerhoff (2002).

Como proposta para simplificar essa classificação, usando Coston (1998) e Furneaux e Ryan (2014), produzimos o seguinte esquema:

\section{FIGURA 2 CLASSIFICAÇÃO DAS PARCERIAS OSCS-GOVERNO}

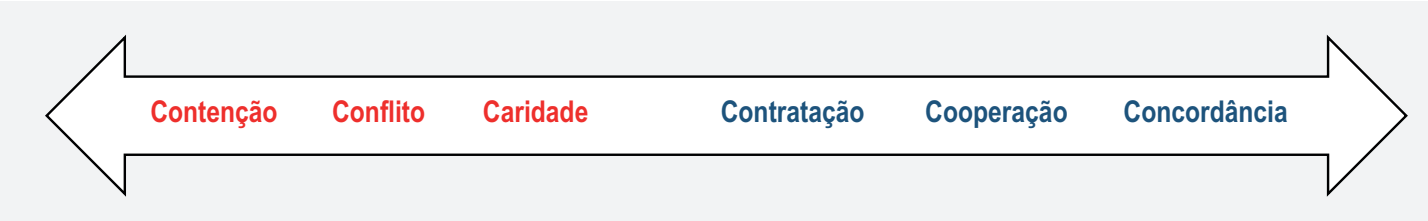

Fonte: Elaborado pelos autores.

A Figura 2 resume os tipos ideais de parcerias, a parte inicial de uma tipologia de acordo com Doty e Glick (1994). Os tipos em vermelho à esquerda podem ser considerados resistentes à interação do governo e têm menos potencial para produzir resultados efetivos. Por outro lado, os da direita, em azul, têm maior potencial para produzir resultados efetivos e, exceto contratantes, podem ser considerados mais abertos à interação participativa. Os tipos de parceria são descritos abaixo.

Em uma parceria de contenção, o governo procura restringir a autonomia e influência das OSCs, por meio de controles desproporcionais, regulação que limita a operação ou atividade das organizações, ou exigências burocráticas excessivas. Nessa forma de relacionamento, há pouco diálogo e falta de confiança entre as partes. Nenhum valor e objetivo comum é identificado. Pode haver perseguição de organizações dissidentes.

No tipo de conflitos, diferenças importantes são encontradas nos valores e objetivos entre as partes, tornando possível identificar vários sistemas de desempenho, a variação do perfil do profissional e

\footnotetext{
${ }^{1}$ Existem vários modelos de financiamento do governo para OSCs no Brasil: financiamento indireto por meio de isenções fiscais, bem como diversas formas de contratação e concessão de subsídios. Terceirização, baseada em sistemas de vouchers, não é um tipo de relacionamento encontrado no Brasil.
} 
as capacidades organizacionais dos dois lados. A Circulação de informação é difícil. O governo pode encontrar formas de restringir as atividades das OSCs através da regulação, controle burocrático excessivo, mas não de uma forma que impeça que as parcerias ocorram. Espaços de diálogo podem existir, mas não são eficazes para apoiar a aproximação entre governo e OSCs. As OSCs são mais propensas a usar formas de mobilização contenciosa para alcançar influência política. Perseguições abertas contra dissidentes são improváveis, mas os mecanismos de financiamento podem não ser transparentes, não garantindo acesso justo aos recursos.

No tipo de caridade, as definições sobre parcerias no desenho de políticas públicas são baixas ou inexistentes. Os relacionamentos tendem a ser escassos ou com baixo grau de monitoramento e controle pelo governo. A participação e as relações interpessoais tendem a ser mais cerimoniais.

No tipo de contratação destacam-se as orientações e detalhes sobre parcerias no desenho de políticas públicas, como padrões, uso de ferramentas compartilhadas de planejamento e gestão, e maior detalhamento de regulamentação quanto ao funcionamento das parcerias. As contratações podem ocorrer independentemente do alinhamento de valores e objetivos. No entanto, requer capacidades organizacionais mais desenvolvidas, tanto na burocracia pública quanto nas organizações da sociedade civil.

Quanto as relações de cooperação a história da interação entre governo e organizações da sociedade civil ganha importância. Espaços formais e informais de participação e diálogo tendem a funcionar. Existe algum nível de compartilhamento de valores e objetivos, garantindo uma base de confiança para os relacionamentos. Pode haver cooperação mesmo em contratos formais usando lógica burocrática ou de mercado, mas nesse tipo de parceria os fatores relacionais e discricionariedade ganham destaque, seja na solução de conflitos potenciais, seja no aprimoramento do modelo e geração de inovações. Os fatores relacionais dependem dos perfis membros do governo e dos staffs das organizações da sociedade civil, assim como o papel dos líderes na construção dos diálogos.

A concordância ocorre quando as organizações da sociedade civil possuem grande legitimidade e capacidade, capazes de promover sua diferenciação aos olhos dos órgãos públicos com os quais se relacionam. Existe um rígido alinhamento de valores e objetivos, permitindo a redução do controle e foco nos resultados compartilhados. As organizações da sociedade civil tendem a estar altamente presentes em serviços complementares aos ofertados pelo Estado. O Estado considera essas organizações como parte de suas políticas públicas e busca a integração, com a preocupação de construir capacidades conjuntas. Esse tipo de relacionamento requer um alto grau de capacidade organizacional e envolvimento de líderes e dirigentes nos diálogos.

No geral, estudos empíricos aplicando os modelos de parcerias focaram em comparações no nível macro, buscando classificá-los e posicioná-los em uma escala mostrando diferenças de países ou distinções de áreas de políticas públicas (Appe \& Layton, 2016; Bode \& Brandsen, 2014; Buckingham, 2012; Furneaux \& Ryan, 2014; Proulx, Bourque, \& Savard, 2007; Young, 2006).

Observamos que este conjunto de estudos é útil para identificar diferenças e semelhanças entre modelos e representar bons esquemas para testar proposições em distintos contextos. Na maioria dos estudos que detalham esses modelos (Brinkerhoff, 2002; Coston, 1998; Najam, 2000) é possível observar trocas inter-organizacionais: compartilhamento de informações, níveis de interação informal, maior ou menor oportunidades de participação na tomada de decisões, dependência mútua, grau de autonomia, e diferentes formas de compartilhamento de recursos, alinhamento de metas.

A principal limitação do uso de tipologias ou modelos para capturar a diversidade é que eles frequentemente se sobrepõem, fazendo com que o esforço de classificação seja uma aproximação 
imperfeita. Os modelos podem ser melhor descritos e detalhados para identificar e garantir que, mesmo com sobreposições, seja possível lidar analiticamente com os achados. Essa etapa, segundo Doty e Glick (1994), visa apontar e detalhar as múltiplas dimensões que formam os tipos ideais.

Em relação aos níveis de análise, Najam (2000) apontou a necessidade de considerar tanto as características do setor quanto a política; e a racionalidade de cada contexto institucional/ organizacional. Bode e Brandsen (2014) classificam os estudos considerando a microdinâmica das parcerias como um subconjunto separado de estudos. A maioria dos estudos com foco em microdinâmica é baseada em estudos de caso, usando diferentes variáveis e categorias, tornando mais difícil testar e comparar.

A literatura descreve modelos de parcerias sem diferenciar níveis de análise. Propomos fornecer mais detalhes sobre isso, com base em dois níveis analíticos: o contexto político-institucional e a dinâmica microorganizacional e sua integração para uso na classificação dos modelos de parceria.

Variáveis ligadas ao contexto político-institucional são listadas:

1. História da interação: muitos estudos apontam para os efeitos da trajetória de dependência. Nesse sentido, uma história de maior aproximação no contexto específico do campo de políticas públicas favorece a construção de parcerias mais colaborativas (Mcloughlin, 2011).

2. Vantagens comparativas: estudos que buscam realizar uma análise econômica das parcerias, analisam as vantagens comparativas das formas de provisão e suas interações (Hansmann, 1987; Lipsky \& Smith, 1990, Salamon 1995, Young, 2000). A existência de vantagens comparativas que promovam a eficiência, a diversificação ou aumentem a qualidade dos serviços prestados por organizações da sociedade civil pode facilitar as colaborações.

3. Abertura do governo: Maior colaboração pode ser obtida através da existência de espaços de participação institucionalizados. O uso de outras formas de influência contenciosa pelas organizações da sociedade civil no contexto da área de política pública pode limitar a colaboração ou, quando combinadas a outras estratégias, contribuir para influenciá-la positivamente.

4. Regulamentação: Trabalhos mais recentes que buscam atualizar os modelos de Coston e Najam (Almog-Bar \& Zychlinski, 2012; Brown \& Troutt, 2004; Furneaux \& Ryan, 2014; Proulx et al., 2007) têm identificado cada vez mais questões regulatórias. Fatores regulatórios que visam promover a transparência e a concorrência leal devem ser permitidos. Fatores regulatórios aumentam o controle burocrático sobre as organizações da sociedade civil ou em casos que contenham normas ambíguas ou normas inexistentes que dificultem a implementação de parcerias devem ser desativados.

As variáveis que vem mediando as relações interorganizacionais são:

5. Autonomia de organizações da sociedade civil: Na questão do fluxo de recursos e dependência versus autonomia de organizações da sociedade civil, muitos estudos procuraram detalhar e diferenciar as formas que afetam as parcerias (Johansson, 2003; Mcloughlin; 2011; Mendonça \& Araújo, 2011; Ramanath, 2009). Dependência de recursos e autonomia são fatores relacionais e precisam ser sistematicamente acessados em cada contexto (McLoughlin, 2011) considerando os perfis das organizações da sociedade civil envolvidas.

6. Alinhamento do objetivo: Mesmo que as ações sejam complementares, internamente, se não houver correspondência sobre o papel da parceria, os objetivos tendem a não estar alinhados (Albrecht, 
2018). Brinkerhoof (2002) refere-se à mutualidade e identidade organizacional. A mutualidade é alta quando o nível de interdependência implica integração com valores comuns definidos coletivamente. Identidade organizacional refere-se à manutenção da autonomia e distinção de uma determinada organização.

7. Liderança e interações pessoais: estas são deixadas de fora da maioria dos modelos (Proulx et al., 2007), mas foram identificadas como tendo um impacto nas parcerias (Almog-Bar \& Zychlinski, 2012; Brown \& Troutt, 2004; Shaw, 2003).

8. Capacidade do Governo: Refere-se a procedimentos administrativos e ferramentas de gestão desenvolvidos ou adaptados para apoiar parcerias. Alguns deles devem incluir a dimensão de parcerias nas fases de planejamento e avaliação de políticas públicas, usando indicadores de resultados específicos; fornecimento de sistemas de informação adaptados para lidar com as especificidades deste tipo de relacionamento; formação de gestores públicos. Apesar disso, a existência de diretrizes ou políticas gerais ou setoriais que possam orientar e apoiar a prestação de serviços por meio de parcerias com organizações da sociedade civil ainda é rara (Brandsen \& Pappe, 2015).

A Figura 3 resume o modelo analítico proposto neste estudo: Quando, após a análise, a classificação dos fatores da relação é posicionada à esquerda, a tendência é que o tipo de parceria seja Contenção, Conflito ou Caridade. Quando os fatores são posicionados à direita, a tendência é que o tipo de parceria seja Contratação, Cooperação ou Concordância, seguindo o intervalo mostrado na Figura 2. Este modelo analítico permite comparação entre os tipos de relacionamentos. Por meio da comparação, foi possível obter detalhes sobre os diferenciais de cada caso, dependendo de contextos específicos.

\section{FIGURA 3 CONTEXTO INSTITUCIONAL DA POLÍTICA PÚBLICA}

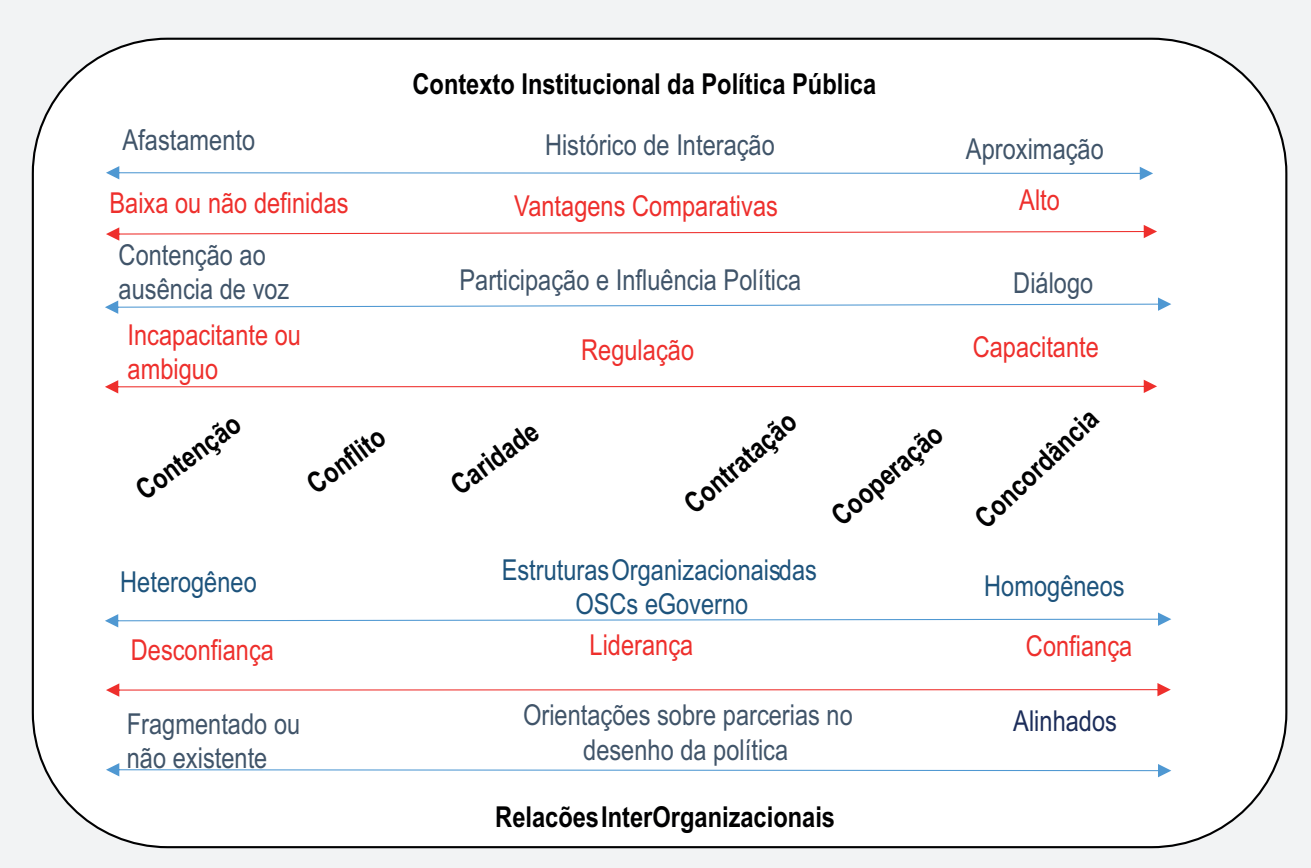

Fonte: Elaborado pelos autores. 


\subsection{Parceria no contexto da AIDS, assistência social e cultura}

\subsubsection{AIDS}

A política de AIDS no Brasil foi elaborada pela primeira vez na década de 1980, durante o período de redemocratização do país. Vários movimentos sociais ressurgem exigindo direitos e o Estado assume a responsabilidade pela provisão de bem-estar social (Galvão, 2000).

Diferentes atores participaram desses movimentos: profissionais de saúde do movimento de reforma sanitária, grupos LGBT, artistas e ativistas pró-democracia que foram diagnosticados com HIV e manifestaram a síndrome durante a epidemia (Campos et al., 2012). Essas mobilizações levaram à criação de organizações da sociedade civil (chamadas "ONGs da AIDS") e abordaram demandas mais amplas expressas em saúde e outras áreas sociais, conectando os princípios do Sistema Único de Saúde (SUS) ao Programa de AIDS do país: universalidade, descentralização e integração. A comunidade internacional reconheceu o Programa Brasileiro de Aids como um exemplo de sucesso (Parker, 2003; Teixeira, 1997).

Quatro acordos entre o governo federal e o Banco Mundial contribuíram para o processo de consolidação e financiamento dessa política. Esses acordos permitiram o financiamento específico de organizações da sociedade civil por meio de recursos captados através de organizações internacionais, sem envolver diretamente os procedimentos da administração pública. Isso proporcionou um meio mais flexível de implementar projetos, eliminando a necessidade de submeter-se a regras estritas e os custos de engajar a burocracia estatal (Campos, 2008).

Dentro dessa política, há uma definição clara de papéis para as organizações da sociedade civil e para o Estado: organizações da sociedade civil que promovem a prevenção, monitoram e apoiam a garantia dos direitos das pessoas vivendo com HIV e grupos vulneráveis; e o Estado provendo tratamento e assistência de saúde especializada. Mais recentemente, o foco passou a aplicar os testes rápidos de HIV realizados por organizações da sociedade civil fora de um ambiente de laboratório, as chamadas 'testagem por pares' (Brasil, 2015).

Antes da implementação do terceiro convênio com o Banco Mundial, o Ministério da Saúde pressionou por uma implementação e integração mais descentralizada com o SUS (Campos, 2008; UNESCO, 2005). A política foi assim descentralizada para os níveis estadual e municipal. Ferramentas de planejamento e mecanismos específicos de financiamento foram criados para garantir a disponibilidade de recursos para organizações da sociedade civil nos níveis subnacionais.

Organizações da sociedade civil continuaram desempenhando um papel amplo dentro da política de AIDS. Essas organizações participam ativamente de iniciativas como a Comissão de Engajamento de Movimentos Sociais (focada na prestação de contas das relações entre entidades sem fins lucrativos); a Comissão Nacional de Doenças Sexualmente Transmissíveis, AIDS e Hepatites Virais (enfocando aspectos técnicos relacionados ao controle de doenças); e várias Câmaras Técnicas do Departamento de DST / Aids (espaços participativos na estrutura do Ministério da Saúde que envolvem usuários, universidades e especialistas).

\subsubsection{Assistência Social}

As relações Estado-sociedade civil no campo da assistência social no Brasil sempre foram pouco claras, em um misto de complementaridade e subsidiariedade, entre o dever do Estado e a filantropia privada. Essa falta de clareza gera ambiguidades e ambivalências na oferta de bens e serviços públicos a grupos 
de risco e socialmente vulneráveis, como os das pessoas com deficiência, crianças e adolescentes, idosos e pessoas em situação de rua (Araújo, 2017; Mestriner, 2001).

Seguindo as disposições da Constituição de 1988 e outras regulamentações relativas à assistência social no Brasil, o Sistema Único de Assistência Social (SUAS) foi implementado em 2006. O Estado, portanto, procura colocar em prática a política pública de assistência social de modo universal, na qual o governo tem um papel central oferecendo diretrizes, bem como, fornecendo serviços de modo direto (Sposati, 2012). A implementação do SUAS tendo a centralidade no Estado criou profundas tensões e conflitos com organizações da sociedade civil que vinham trabalhando anteriormente em por meio de métodos religiosos / filantrópicos.

O SUAS avançou em relação às diretrizes técnicas, estabelecendo novas bases para o relacionamento entre o Estado e organizações da sociedade civil como prestadores de serviços complementares. Estes últimos tornaram-se não apenas prestadores de serviços, mas co-gestores participando de conselhos locais de assistência social e sendo co-responsáveis por garantir os direitos sociais (Araújo, 2015; Brasil, PNAS / NOBAS, 2004).

O financiamento de organizações da sociedade civil para fornecer assistência social foi disponibilizado por meio de financiamento indireto, como isenções de impostos e financiamento direto (subsídios). O financiamento indireto exigiu um complexo processo burocrático envolvendo múltiplos registros e sistemas de certificação que muitas vezes dependem de conexões das organizações da sociedade civil com as casas legislativas.

A efetiva integração de organizações da sociedade civil no SUAS é um dos principais desafios para as parcerias (Araújo, 2015b, 2017). Novos parâmetros e padrões em relação aos serviços foram introduzidos, demandando das organizações da sociedade civil exigiram mais integração com o Sistema.

\subsubsection{Cultura Viva}

Com o famoso cantor e compositor Gilberto Gil como ministro da Cultura em 2003, uma nova era começou para as políticas culturais no Brasil. Buscando reverter a concentração regional e temática causada pelas políticas anteriores orientadas para mercado, o programa Cultura Viva foi proposto em 2004 para promover organizações culturais que haviam sido excluídas dos sistemas tradicionais de financiamento (Turino, 2009).

O Cultura Viva foi concebido para fortalecer as práticas culturais existentes, promovendo a produção e circulação da expressão cultural da natureza local e comunitária. A estrutura chave para a implementação desta política são os Pontos de Cultura.

O programa busca apoiar, melhorar e promover atividades que organizações da sociedade civil já patrocinam em suas comunidades locais, principalmente em locais remotos, apoiando, assim, diferentes expressões culturais e artísticas em todas as regiões do país. O Estado reconhece as iniciativas dessas entidades sem fins lucrativos em suas comunidades e oferece-lhes o apoio institucional e financeiro necessário para a operação, melhoria e expansão de suas iniciativas. Essas organizações da sociedade civil foram nomeadas Pontos de Cultura.

Em muitas dessas organizações, artistas ou jovens líderes gerenciam coletivos culturais, e o Ponto de Cultura foi a primeira parceria que estabeleceram com o Estado. Não houve experiência anterior em questões de gestão, especialmente no que diz respeito à execução de contratos e transferência de recursos públicos. 
É importante destacar o quão diversas são as organizações que se tornam Ponto de Cultura, para evitar generalizações. No entanto, compartilham um elemento comum: são organizações que operam em redes, já que o programa Cultura Viva é projetado para atividades em rede. Assim, os Pontos de Cultura influenciam uns aos outros, incluindo aqueles que não recebem recursos diretamente do programa de governo (Medeiros et al., 2015).

Apesar de sua proposta inovadora na implementação de políticas mais horizontais, o desenho de políticas não criou ferramentas regulatórias e de gestão adequadas para lidar com perfis específicos e diversificados de organizações da sociedade civil culturais, como treinamento, diretrizes e sistemas de monitoramento. À medida que essas organizações são submetidas às regras burocráticas do governo, elas enfrentam desafios e mudanças na maneira de agir (Medeiros, 2013).

Diversas organizações participantes se mobilizaram e pressionaram pela mudança de procedimentos, tendo sucesso em propor um projeto de lei ao Congresso, que foi aprovado por meio da Lei ${ }^{\circ}$ 13018/2014. No entanto, a lei ainda carece de alguns detalhes, definições e regulamentações na burocracia do Estado para ser adequadamente implementada.

\subsection{Análise dos modelos de relacionamento nas áreas de Políticas de AIDS, Assistência Social e Cultura}

O modelo analítico explicado nas Figuras 2 e 3 foi utilizado para resumir os três casos no Quadro 2 abaixo. Utilizamos os dados descritos no Quadro 1 da metodologia.

Fizemos um esforço para entender o que leva uma parceria a um espectro mais colaborativo ou mais conflitante, como mostra a Figura 2. Para tanto, delimitamos dois tipos de fatores, um relacionado ao contexto político-institucional e outro ao micro-organizacional, como mostra a Figura 3. A comparação visual dos três casos no Quadro 2 é facilitada, com os destaques em vermelhos representando modelos mais conflitantes e os azuis, os mais colaborativos. Usamos essa sistematização para discutir os casos separadamente e propor classificações para cada modelo de parceria encontrado.

\section{QUADRO 2 COMPARAÇÃO DE MODELOS DE PARCERIAS}

\begin{tabular}{|c|c|c|c|}
\hline & AIDS & Assistência Social & Cultura Viva \\
\hline \multicolumn{4}{|c|}{ Political-Institutional Context } \\
\hline $\begin{array}{l}\text { 1-Histórico de } \\
\text { Interação }\end{array}$ & $\begin{array}{l}\text { OSCs com papel central } \\
\text { na elaboração e design da } \\
\text { política (Teixeira, 1997; } \\
\text { Parker, 2003; Galvão, 2000). }\end{array}$ & $\begin{array}{l}\text { OSCs como provedores principais } \\
\text { de serviços, mesmo com mudanças } \\
\text { recentes e expansão da provisão } \\
\text { pública e aumento da competição. } \\
\text { (Araújo, 2015a, 2015b, 2017). }\end{array}$ & $\begin{array}{l}\text { Estado tem papel central na criação e } \\
\text { fomento dos bens culturais, juntamente } \\
\text { com o mercado (Medeiros, 2013; } \\
\text { Medeiros et al., 2015). }\end{array}$ \\
\hline $\begin{array}{l}\text { 2-Vantagens } \\
\text { comparativas }\end{array}$ & $\begin{array}{l}\text { Complementaridade } \\
\text { (Campos \& Mendonça,2010; } \\
\text { Campos et al., 2012). }\end{array}$ & $\begin{array}{l}\text { Modelo de competição entre } \\
\text { provisão pública e das OSCs. } \\
\text { (Araújo, 2015a, 2015b, 2017). }\end{array}$ & $\begin{array}{l}\text { Papel de suplementaridade bem } \\
\text { definido. } 0 \text { estado fomenta as } \\
\text { iniciativas culturais da sociedade não } \\
\text { presentes no mainstream das políticas } \\
\text { culturais (Turino, 2009; Medeiros, } \\
\text { 2013; Medeiros et al., 2015). }\end{array}$ \\
\hline
\end{tabular}




\begin{tabular}{|c|c|c|c|}
\hline & AIDS & Assistência Social & Cultura Viva \\
\hline $\begin{array}{l}\text { 3- Abertura do } \\
\text { Governo }\end{array}$ & $\begin{array}{l}\text { Espaços institucionalizados } \\
\text { de participação no Ministério } \\
\text { da Saúde combinado } \\
\text { com outras formas de } \\
\text { ações de contenção pelas } \\
\text { OSCs (dados primários de } \\
\text { entrevistas- Ministério da } \\
\text { Saúde). }\end{array}$ & $\begin{array}{l}\text { Conselho nacional de Assistência } \\
\text { Social (CNAS) como espaço } \\
\text { institucionalizado par OSCs, } \\
\text { usuários e profissionais da } \\
\text { assistência social, os últimos tendo } \\
\text { maior influência. OSCs possuen } \\
\text { ibfluencia e lobby no Confresso } \\
\text { Nacional (Araújo, 2015a, 2017). }\end{array}$ & $\begin{array}{l}\text { Abertura informal, mas não } \\
\text { institucionalizada como espaço } \\
\text { de participação (Medeiros, 2013; } \\
\text { Medeiros et al., 2015). }\end{array}$ \\
\hline 4- Regulação & $\begin{array}{l}\text { Completa, clara e bem } \\
\text { internalizada no sistema de } \\
\text { implementação (Mendonça, } \\
\text { 2018). }\end{array}$ & $\begin{array}{l}\text { Detalhada e considerada confusa } \\
\text { pelas OSCs. Impõe exigências } \\
\text { burocráticas (Mendonça, 2018; } \\
\text { Araújo, 2015a, 2017). }\end{array}$ & $\begin{array}{l}\text { Regulação ambígua e incompleta, } \\
\text { pode comprometer a descentralização } \\
\text { e operacionalização (Medeiros et al., } \\
\text { 2015; Mendonça, 2018). }\end{array}$ \\
\hline \multicolumn{4}{|c|}{ Micro-Dinâmicas Organizacionais } \\
\hline $\begin{array}{l}\text { 5- Autonomia } \\
\text { das OSCs }\end{array}$ & $\begin{array}{l}\text { Politicamente engajadas } \\
\text { e bem estruturadas, as } \\
\text { OSCs têm capacidades } \\
\text { técnicas bem desenvolvidas. } \\
\text { Possuem múltiplas fontes de } \\
\text { financiamento. }\end{array}$ & $\begin{array}{l}\text { OSCs financiadas } \\
\text { predominantemente pelo Estado. } \\
\text { Poucas profissionalizadas } \\
\text { conseguem lidar com a } \\
\text { dependencia de recursos. }\end{array}$ & $\begin{array}{l}\text { Predominancia de OSCs pequenas } \\
\text { com estruturas informais. Mesmo no } \\
\text { pequeno periodo de implementação } \\
\text { houve impacto no enfraquecimeto da } \\
\text { autonomia das OSCs no Cultura Viva } \\
\text { (Medeiros et al., 2015). }\end{array}$ \\
\hline $\begin{array}{l}\text { 6- Alinhamento } \\
\text { de Objetivos }\end{array}$ & $\begin{array}{l}\text { Alto grau de alinhamento de } \\
\text { objetivos. }\end{array}$ & $\begin{array}{l}\text { Baixo alinhamento. Logica do Estado } \\
\text { baseada em objetivos amplos da } \\
\text { política pública, lógica das OSCs } \\
\text { predominantemente focada na } \\
\text { filantropia, caridade e valores } \\
\text { religiosos (Sposati, 2008, 2009, } \\
\text { 2012). }\end{array}$ & $\begin{array}{l}\text { Baixa capacidade de implementação } \\
\text { no nível central. Descentralização } \\
\text { difícil- sem diferenciação de } \\
\text { responsabilidades entre os níveis } \\
\text { subnacionais (Medeiros, 2013; } \\
\text { Medeiros et al., 2015). }\end{array}$ \\
\hline 7- Liderança & $\begin{array}{l}\text { OSCs e profissionais de } \\
\text { saúde com laços fortes } \\
\text { (Campos et al., 2012). }\end{array}$ & $\begin{array}{l}\text { Presença forte de politicosStrong } \\
\text { presence of politicians representing } \\
\text { interests of nonprofits (primary } \\
\text { data- Interview at Minister of Social } \\
\text { Development) }\end{array}$ & $\begin{array}{l}\text { Suporte de líderes do programa no } \\
\text { Ministério da Cultura (dados primários } \\
\text { de entrevistas- Ministério da Cultura; } \\
\text { Medeiros, 2013) }\end{array}$ \\
\hline $\begin{array}{l}\text { 8- Capacidade } \\
\text { Governamental }\end{array}$ & $\begin{array}{l}\text { Parcerias consideradas no } \\
\text { planejamento e avaliação } \\
\text { de instrumentos de políticas } \\
\text { públicas (Campos, 2008; } \\
\text { UNESCO, 2005). }\end{array}$ & $\begin{array}{l}\text { Aplicação difícil de serviços } \\
\text { tipificados e outros instrumentos de } \\
\text { gestão criados pelo SUAS (Araújo, } \\
\text { 2015a). }\end{array}$ & $\begin{array}{l}\text { Proposição inovadora de estrturas } \\
\text { em rede, ainda que de dificil } \\
\text { operacionalização (Medeiros, 2013; } \\
\text { Medeiros et al., 2015). }\end{array}$ \\
\hline
\end{tabular}

Fonte: Elaborado pelos autores.

No caso da AIDS há uma convergência de objetivos compartilhados e estratégias entre as OSCs e o Estado, presente desde a mobilização que delimitaram a agenda inicial da política na década de 80. Os papéis estão claramente definidos: o Estado prove assistência e tratamento para os infectados com HIV, enquanto as OSCs realizam prevenção e defesa de direitos. Entre os três casos estudados, este demostra maior vantagem comparativa, com as OSCs de AIDS desempenhando funções que não são facilmente realizáveis pelo estado. 
Existem muitos diálogos e espaços de participação e diferentes áreas do Ministério da Saúde, com diversos meios de consulta e produção de conhecimento compartilhado. Os profissionais de saúde no setor público possuem maior envolvimento com movimentos socais, e esta área também demostra melhores capacidades administrativas e gestão entre as três estudadas. O programa de AIDS, em particular, apresenta um desenho de política compartilhado que inclui as parcerias de maneira central desde sua formulação. É possível encontrar especificações no planejamento, no compartilhamento técnico, no estabelecimento de objetivos e indicadores aspectos relacionados às parcerias.

As OSCs nesta área acessam outros tipos de fundo, que não apenas os públicos estatais, especialmente recursos advindos da cooperação internacional nos acordos de cooperação como Banco Mundial e outras entidades internacionais. Estes atores internacionais também investiram na construção de capacidades das OSCs. Isto permitiu que as organizações desta área mantivessem sua autonomia e influência na política, desenvolvendo relacionamentos mais orgânicos como Estado, incluindo o constante fluxo de profissionais, engajamento ativo em diferentes instancias no Ministério da Saúde/ Departamento de AIDS. Esta combinação de complementaridade, histórico de diálogo e aproximação e boa coordenação posiciona estas parcerias no modelo de cooperação.

No caso da assistência social, mudanças recentes na política aumentaram os níveis de tensão nas relações. A provisão estatal foi fortalecida, causando concorrência, em um modelo que anteriormente poderia ser caracterizado como caridade. As mudanças também implicaram conflitos sobre estratégias de trabalho. As OSCs operavam sob formas mais paternalistas, enquanto o Estado operava de forma a salvaguardar os direitos das pessoas dentro de um quadro universalista. As tensões também aumentaram quando regras mais estritas para receber financiamento público indireto entraram em vigor (principalmente através de deduções fiscais e isenções).

O Ministério do Desenvolvimento Social experimentou uma crescente profissionalização no período analisado. Nesse caso, o impacto nas parcerias foi negativo, com as OSCs encarando os padrões de serviços e outras diretrizes como pressões para o seu funcionamento. Este é, portanto, um modelo com características de conflito e de Contratação.

Quanto à cultura, o caso observado foi um novo programa com características únicas jamais vistas nas políticas culturais brasileiras, com muitas inovações não apenas em metas, mas também em estratégias, como a promoção de redes e formas mais horizontais de prestação de serviços. O novo programa beneficiou-se da liderança do ministro Gilberto Gil e de sua equipe técnica. A liderança também foi importante na construção de diálogos e alinhamento com organizações culturais. Há um alinhamento de metas, já que o Estado busca reconhecer e promover iniciativas culturais locais, suas singularidades, em um desenho suplementar de provisão, ou seja, o estado não era capaz de produzir serviços sem as OSCs.

No entanto, o Ministério da Cultura não dispunha de recursos para colocar em prática todos os objetivos. A política ainda precisa melhorar sua regulamentação, a fim de permitir que o Ministro da Cultura atue com menos burocracia no controle de processos. Apesar da organização de eventos e da promoção de redes, as OSCs não têm espaços formais de participação para influenciar políticas, como é possível observar em outras áreas. As OSCs influenciam a política de maneira menos formalizada, criando coalizões, mas de forma incipiente, devido à heterogeneidade das organizações no campo.

As OSCs receberam inicialmente o Cultura Viva com entusiasmo. No entanto, a tentativa do governo de apoiar o trabalho das OSCs resultou em cooptação, já que essas organizações se submetem a demandas burocráticas a tal ponto que suas outras atividades são comprometidas. Assim, estas parcerias demostraram aspectos mistos de modelos de contratação e conflito. 


\section{CONSIDERAÇÕES FINAIS}

A literatura sobre parcerias enfoca a grande diversidade de OSCs e modelos de parceria com o estado para a prestação de serviços (Coston, 1998; Brinkerhoof, 2002; Najam, 2000; Young, 2000, 2006). A maioria dos estudos empíricos tendem a fazer esforços para categorizar parcerias, geralmente comparando países ou áreas de políticas públicas em um nível macro (Appe \& Layton, 2016; Bode \& Brandsen, 2014; Buckingham, 2012; Furneaux \& Ryan, 2014; Proulx et al., 2007; Young, 2006).

Este estudo procurou contribuir para o uso desses modelos para entender as diferenças entre diferentes políticas públicas. Ao fazê-lo, introduziu variáveis de nível de campo e variáveis organizacionais para diferenciar melhor os modelos e identificar sobreposições.

Através do uso desses modelos buscamos compreender as diferenças entre as parcerias nas diferentes políticas públicas. Ao fazê-lo, introduzimos variáveis de nível de campo e variáveis organizacionais para diferenciar melhor os modelos e identificar sobreposições.

Comparamos parcerias nas áreas de AIDS, assistência social e cultura. Diferenças entre eles nos ajudaram a testar mais variáveis relacionais para produzir análises usando os modelos.

As abordagens que destacam o nível de campo e os recursos organizacionais são importantes para entender por que um modelo é implementado em vez de outros. Diferentes modelos podem ser identificados. Para a política de aids, prevalece o modelo de cooperação. Para assistência social, identificamos um ambiente de mudança que combina características de conflito e contratação, que evoluiu de um formato de caridade anterior. Um modelo de conflito prevalece e cresce à medida que as mudanças nas políticas são implementadas. Para a cultura, o modelo predominante é ambíguo e apresenta características de contratação e conflito.

Portanto, esses modelos nunca são puros e não podemos dizer de antemão que a cooperação é a forma ideal de parceria. Esta seria uma sugestão primária para futuras pesquisas. A cooperação leva a maior eficiência, melhor qualidade de serviços, eficácia e integração com a política? Além disso, quais dos elementos que permitem a parceria levam a modelos mais cooperativos ou de confronto? Esta é a agenda apresentada para pesquisas futuras.

Neste estudo, procuramos complementar a análise de modelos de parceria (Najam, 2000; Young, 2000) com outros elementos, como o perfil das organizações sem fins lucrativos e seus profissionais, tipos de financiamento, espaços de engajamento e elementos que foram analisados em outros estudos (Buckingham, 2012; Furneaux \& Ryan, 2014; Proulx et al., 2007).

Acreditamos que pesquisas futuras focadas nessa agenda podem combinar abordagens qualitativas e quantitativas com pesquisas sobre elementos específicos das parcerias e características das OSCs. Eles também podem testar a variação em modelos com as mesmas áreas de políticas distribuídas para diferentes contextos locais de implementação e nível governamental.

Em relação às implicações práticas, os resultados desta pesquisa apoiam a recomendação de esforços para avançar uma agenda subnacional sobre parcerias que possam combinar diretrizes gerais com especificidades de áreas políticas. As parcerias devem ser vistas como instrumentos de política pública, lembrando continuamente que a ação pública vai muito além do estado. 


\section{REFERÊNCIAS}

Albrecht, K. (2018). Institutional logics and accountability: Advancing an integrated framework in nonprofit - public partnerships. Journal of Public and Nonprofit Affairs, 4(3), 284-305. Recuperado de https://doi.org/10.20899/jpna.4.3.284-305

Almog-Bar, M., \& Zychlinski, E. (2012). A Façade of Collaboration: Relationships between philanthropic foundations and the government in social policymaking in Israel. Public Management Review, 14(6), 795-814. Recuperado de https://doi.org/10.1080/14 719037.2011 .642625

Alves, M. A., \& Koga, N. M. (2006). Brazilian nonprofit organizations and the new legal framework: an institutional perspective. $B A R$ - Brazilian Administration Review, 3(2), 68-83. Recuperado de https://dx.doi.org/10.1590/S180776922006000200006

Appe, S., \& Layton, M. (2015). Government and the Nonprofit Sector in Latin America. Nonprofit Policy Forum, 7(2), 117-135. Recuperado de http://doi. org/10.1515/npf-2014-0028

Araújo, E. T. (2015a). Documento técnico contendo levantamento e análise das orientações técnicas, documentação e normativas do CNEAS; bem como padronização de conceitos e conteúdos inerentes aos serviços programas, projetos e benefícios socioassistenciais. Projeto BRA12/006 MDS, Pnud. Brasília, DF: MDS.

Araújo, E. T. (2015b). Documento técnico contendo proposta de Manual de Orientação aos Gestores da Política de Assistência Social no que concerne ao Cadastro Nacional de Entidades de Assistência Social (CNEAS). Projeto BRA12/006 MDS, Pnud. Brasília, DF: MDS.

Araújo, E. T. (2015c). Avaliação do processo de implementação dos Centros-dia de Referência para a Pessoa com Deficiência e suas Famílias, no âmbito do Plano Viver Sem Limite, na Região Nordeste. Relatório Final de Pesquisa. Projeto apoiado pela chamada pública MCTI-CNPq/MDS-SAGI No 24/2013 - DESENVOLVIMENTO SOCIAL, no TEMA 1: Assistência Social - 1.6 A Implementação do Plano Viver Sem Limites. Brasília, DF: MDS.

Araújo, E. T. (2017). Gestão Social e a Política Pública de Assistência Social. Curso gestão social. In J. M.
Oliveira Neto, \& J. T. Silva Jr. (Org). Fortaleza, CE: Fundação Demócrito Rocha/UANE/BID/STDS-Ce. (Curso em 12 Fascículos).

Bode, I., \& Brandsen, T. (2014). State-third Sector Partnerships: A short overview of key issues in the debate. Public Management Review, 16(8), 10551066. Recuperado de https://doi.org/10.1080/1471 9037.2014.957344

Boullosa, R. F. (2013). O SUAS e a Proteção Social Especial para a Pessoa com Deficiência: um caso de inovação em políticas públicas? In Anais do 180 Congreso CLAD sobre la Reforma del Estado y de la Administración Pública, Montevideo, Uruguay.

Brandsen, T. \& Pape, U. (2015). The Netherlands: The Paradox of Government-Nonprofit Partnerships. Voluntas: International Journal of Voluntary and Nonprofit Organizations, 26(6), 2267-2282. Recuperado de https://doi.org/10.1007/s11266015-9646-3

Brasil (2004). Política Nacional de Assistência Social (PNAS), Resolução CNAS, $n^{\circ}$ 145/2004. CNAS: Brasília, DF. Recuperado de https://www.mds.gov. br/webarquivos/publicacao/assistencia_social/ Normativas/PNAS2004.pdf

Brasil (2009). Tipificação Nacional dos Serviços Socioassistenciais. Texto da Resolução no 109, de 11 de novembro de 2009. Brasília, DF. Recuperado de https://www.mds.gov.br/webarquivos/publicacao/ assistencia_social/Normativas/tipificacao.pdf

Brasil. Ministério da Cultura (2013). Programa Arte Cultura e Cidadania - Cultura Viva. Brasília, DF. Recuperado de http://www.cultura.gov.br/ culturaviva/cultura-viva/.

Brasil. Ministério da Saúde (2015). Projeto Brasil Aids-SUS. P3540/LN7901-BR. Ministério da Saúde Secretaria de Vigilância em Saúde Departamento de DST, Aids e Hepatites Virais: Brasília, DF.

Brinkerhoff, J. (2002). Government-nonprofit partnership: a defining framework. Public Administration and Development, 22(1), 19-30.

Brown, L. K., \& Troutt, E. (2004). Funding Relations between Nonprofits and Government: A Positive Example. Nonprofit and Voluntary Sector Quarterly, 33(1), 5-27. Recuperado de https://doi. org/10.1177/0899764003260601 
Buckingham, H. (2012). Capturing Diversity: A Typology of Third Sector Organizations' Responses to Contracting Based on Empirical Evidence from Homelessness Services. Journal of Social Policy, 41(3), 569-589. Recuperado de https://doi. org/10.1017/S0047279412000086

Campos, L. C. M. (2008). ONGs/AIDS: acesso a fundos públicos e sustentabilidade de ações. RAE Revista de Administração de Empresas, 48(3), 81-93. Recuperado de http://dx.doi.org/10.1590/S003475902008000300008

Campos, L. C. M., \& Mendonça, P. (2010). Provisão compartilhada de Serviços Públicos: configuração e desafios a partir da análise da Política Nacional de Combate ao HIV/Aids. Revista Economia \& Gestão, 10(23), 100-117.

Campos, L. C. M., Mendonça, P. M., \& Alves, M. A. (2012). From dot.org to dot.gov: Professional Crossings in the Brazilian National Policy on HIV/ AIDS. Voluntas: International Journal of Voluntary and Nonprofit Organizations, 23(1), 236-256. Recuperado de https://doi.org/10.1007/s11266011-9195-3

Coston, J. M. (1998). A Model and Typology of Government - NGO Relationship. Nonprofit and Voluntary Sector Quarterly, 27(3), 358-382. Recuperado de http://dx.doi. org/10.1177/0899764098273006

Doty, D., \& Glick, W. (1994). Typologies as a Unique Form of Theory Building: Toward Improved Understanding and Modeling. The Academy of Management Review, 19(2), 230-251. Recuperado de http://www.jstor.org/stable/258704

Eisenhardt, K. (1989). Building Theories from Case Study Research. The Academy of Management Review, 14(4), 532-550. Recuperado de http://www. jstor.org/stable/258557

Furneaux, C., \& Ryan, N. (2014). Modelling NPO - Government Relations: Australian case studies. Public Management Review, 16(8), 1113-1140, Recuperado de https://doi.org/10.1080/14719037. 2014.895030

Galvão, J. (2000). AIDS no Brasil: a agenda de construção de uma epidemia. Rio de Janeiro: ABIA; São Paulo, SP: Ed. 34.
Hansmann, H. (1987). Economic theories of nonprofit organization. In W. W. Powell (Ed.). The Nonprofit Sector: A Research Handbook. New Harlem, NY: Yale University Press.

Johansson, S. (2003). Independent Movement or Government Subcontractor? - Strategic Responses of Voluntary Organizations to Institutional Processes. Financial Accountability \& Management, 19(3), 209224. Recuperado de https://doi.org/10.1111/14680408.00171

Lacoumes, P., \& Le Galés, P. (2012). Sociologia da Ação Pública. Tradução e estudo introdutório: George Sarmento, Maceió, AL: EDUFAL.

Lipsky, M., \& Smith, S. (1989). Nonprofit Organizations, Government, and the Welfare State. Political Science Quarterly, 104(4), 625-648. Recuperado de http://www.jstor.org/stable/2151102 Lopez, F. G., \& Abreu, R. (2014). A Participação das ONGs nas Políticas Públicas: o ponto de vista dos gestores federais. IPEA, Textos para Discussão 1949. Brasília, DF: IPEA. Recuperado em 28 de Abril de 2014. Recuperado de http:// www.ipea.gov.br/portal/index.php?option $=\mathrm{com}_{-}$ content\&view $=$ article\&id $=22088$

Lopez, F., Lopes, L. F., Souto, B. \& Sant'Ana, D. (2014). A interação entre organizações da sociedade civil e governo federal: Colaboração nas políticas públicas e aperfeiçoamentos regulatórios possíveis. Brasil em desenvolvimento - Estado planejamento e políticas públicas. v. 2. Brasília, DF: Ipea. Recuperado de http://www.secretariageral.gov.br/atuacao/ mrosc/artigos/brasil-em-desenvolvimento-2014ipea.pdf

Mcloughlin, C. (2011). Factors Affecting State - Non governmental organizations relations in service provision: key themes from the literature. Public Administration and Development, 31(4), 240-251. Recuperado de https://doi.org/10.1002/pad.611

Medeiros, A. K., Alves, M. A., \& Farah, M. F. S. (2015). Programa Cultura Viva e o campo organizacional da cultura: análise de políticas públicas pela perspectiva institucionalista. Revista de Administração Pública, 49(5), 1215-1235. Recuperado de https://dx.doi. org/10.1590/0034-7612130337

Medeiros, A. K. (2013). Políticas públicas e organizações culturais: o caso do programa 
Cultura Viva (Dissertação de Mestrado). Escola de Administração de Empresas de São Paulo, Fundação Getulio Vargas, São Paulo. Recuperado de http://hdl. handle.net/10438/10653

Mendonça, P. M., \& Araujo, E. T. (2011). Sustentabilidade organizacional em ONGs: os casos do Gapa-BA e do Grupo Brasil a partir das contribuições da Teoria da Dependência de Recursos. Revista Gestão \& Planejamento, 12(1), 112-132. Recuperado de https://revistas.unifacs.br/ index.php/rgb/article/view/1120

Mendonça, P. M. E. (2018). Paradigms of Public Management and the Historical Evolution of State CSO Partnerships: A Comparison of AIDS, Social Assistance, and Cultural Policy. In A. B. Savignon, L. Gnan, A. Hinna, \& F. Monteduro (Org.). Studies in Public and Non-Profit Governance. (Vol. 6, 1. ed., pp. 111-130) Bingley: Emerald Publishing Limited.

Mestriner, M. L. (2001). O Estado entre a filantropia e a assistência social. São Paulo, SP: Cortez.

Najam, A. (2000). The Four C's of Government Third Sector-Government Relations. Nonprofit Management and Leadership, 10(4), 375-396. Recuperado de https://doi.org/10.1002/nml.10403

Parker, R. (2003). Construindo os alicerces para a resposta ao HIV/AIDS no Brasil: o desenvolvimento de políticas sobre o HIV/AIDS, 1982-1996. Divulgação em Saúde para Debate. Londrina, PR: Centro Brasileiro de Estudos da Saúde.

Peci, A., Figale, J. \& Sobral, F. (2011). The Invasion of Manufactured Civil Society: government-nonprofit partnerships in a Brazilian state. Public Admin. Dev., 31(5), 377-389. Recuperado de https://doi. org/10.1002/pad.603

Proulx, J., Bourque, D., \& Savard, S. (2007). The Government-Third Sector Interface in Québec. Voluntas: International Journal of Voluntary and Nonprofit Organizations, 18(3), 293-307. Recuperado de https://doi.org/10.1007/s11266007-9045-5

Ramanath, R. (2007). Limits to Institutional Isomorphism: Examining Internal Processes in NGO - Government Interactions. Nonprofit and Voluntary Sector Quarterly, 38(1), 51-76. Recuperado de https://doi.org/10.1177/0899764008315181
Resende, G. A., \& Teodósio, A. S. (2014). Democratização de políticas sociais no Brasil: venturas e desventuras das organizações da sociedade civil. Civitas: Revista de Ciências Sociais, 14(1), 177-192. Recuperado de http://dx.doi. org/10.15448/1984-7289.2014.1.10689

Salamon, L. M. (1995). Partners in Public Service: Government - Nonprofit Relations in the Modern Welfare State. Baltimore, US: The Johns Hopkins University Press.

Salinas, N. S. (2013). Modelos de controle das parcerias entre Estado e entidades do terceiro setor e desenho institucional das políticas públicas. Revista de Direito do Terceiro Setor, 7(14), 9-28.

Shaw, M. M. (2003). Successful collaboration between the nonprofit and public sectors. Nonprofit Management and Leadership, 14(1), 107-120. Recuperado de https://doi.org/10.1002/nml.24

Sposati, A. (2008). Territorialização e desafios à gestão pública inclusiva: o caso da assistência social no Brasil. In Anais do 13o Congreso Internacional del CLAD sobre la Reforma del Estado y de la Administración Pública, Buenos Aires, Argentina.

Sposati, A. (2009). Modelo brasileiro de proteção social não contributiva: concepções fundantes. In Ministério do Desenvolvimento Social e Combate à Fome (MDS) \& Organização das Nações Unidas para a Educação, a Ciência e a Cultura (UNESCO). Concepção e gestão da proteção social não contributiva no Brasil. Brasília, DF: autor.

Sposati, A. (2012). Desafios do sistema de proteção social. In C. G. Stuchi, R. F. S. Paula, \& R. D. O. Paz (Org.) Assistência Social e Filantropia: cenários contemporâneos. São Paulo, SP: Veras.

Stake, R. E. (1995). The art of case study research. Thousand Oaks, CA: Sage.

Stuchi, C. G; Paula, R. F. S.; Paz, R. D. O. (2012). Assistência Social e Filantropia: cenários contemporâneos. São Paulo, SP: Veras.

Teixeira, P. T. (1997). Políticas Públicas em Aids in Políticas, instituições e Aids: enfrentando a epidemia no Brasil. Rio de Janeiro, RJ: ABIA.

Turino, C. (2009). Ponto de cultura: o Brasil de baixo para cima. (2. ed.) São Paulo, SP: Anita Garibaldi. 
UNESCO. (2005). Response to Aids challenges in Brazil: limits and possibilities. Brasilia, DF: autor.

Young, D. R. (2000). Alternative models of government-nonprofit sector relations: theoretical and international perspectives. Nonprofit and Voluntary Sector Quarterly, 29(1), 149-172. Recuperado de https://journals.sagepub.com/doi/ abs/10.1177/0899764000291009.

\section{Patrícia Maria Emerenciano de Mendonça}

https://orcid.org/0000-0002-5675-4261

Doutora em Administração Pública e Governo pela Escola de Administração de Empresas de São Paulo da Fundação Getulio Vargas (FGV EAESP); Coordenadora do Programa de Mestrado em Gestão de Políticas Públicas na Escola de Artes Ciências e Humanidades da Universidade de São Paulo (EACH-USP).

E-mail: pmendonca@usp.br

\section{Anny Karine de Medeiros}

https://orcid.org/0000-0002-9208-5720

Mestre e Doutoranda em Administração Pública e Governo pela Escola de Administração de Empresas de São Paulo da Fundação Getulio Vargas (FGV EAESP). E-mail: annykmedeiros@gmail.com

\section{Edgilson Tavares de Araújo}

https://orcid.org/0000-0002-2145-8796

Doutor em Serviço Social pela Pontifícia Universidade Católica de São Paulo (PUC-SP); Pós-Doutorando na Universidade de Brasília (UnB) pelo Programa de Pós-Graduação em Desenvolvimento, Sociedade e Cooperação Internacional do Centro de Estudos Avançados Multidisciplinares; Professor da Universidade Federal da Bahia (UFBA); Bolsista de Produtividade em Desenvolvimento Tecnológico e Extensão Inovadora do Conselho Nacional de Desenvolvimento Científico e Tecnológico (CNPq). E-mail: edgilson@gmail.com 